\section{SIMILAR DISTRIBUTION OF SENTINEL LYMPH NODES AND NODAL METASTASES IN CERVICAL AND ENDOMETRIAL CANCER. A PROSPECTIVE STUDY BASED ON LYMPHATIC ANATOMY}

O Lührs*, M Bollino, L Ekdahl, C Lönnerfors, B Geppert, J Persson. Lunds universitet, Faculty of Medicine, LUND, Sweden

\subsection{6/ijgc-2021-ESG0.644}

Introduction/Background* Comparing the anatomical distribution of pelvic sentinel lymph nodes (SLN) in cervical and endometrial cancer.

Methodology Detailed SLN mapping results were prospectively retrieved in cervical $(n=145)$ or high risk endometrial cancer $(n=201)$ patients. Cervically injected Indocyanine Green (ICG), allowing for reinjection, was used as tracer. An anatomically based definition of SLNs was adhered to evaluating the upper (UPP) and lower (LPP) paracervical lymphatic pathways. The positions of SLNs were intraoperatively depicted on an anatomical chart. A completory pelvic lymphadenectomy was performed in all patients and in addition, $\mathrm{xx}$ underwent a paraaortic lympadenectomy. Mapping rates and anatomical distribution of SLNs were compared between groups.

Result(s)* The bilateral mapping rate was $97.9 \%$ and $95.0 \%$ for cervical and endometrial cancer respectively. All pelvic node positive women (cervical cancer $n=19$, endometrial cancer $n=37$ ) had at least one metastatic SLN. The proportion of typically positioned (interiliac and proximal obturator fossa) SLNs along the UPP was similar between groups $(78.1 \%$ vs $82.1 \%, \mathrm{p}=.09$ ) with a similar distribution of SLN metastases; $54.1 \%$ and $48.6 \%$ respectively were located in the obturator fossa. Anatomically typical positions could not be defined along the LPP.

Conclusion* A cervical injection of ICG results in similar anatomical distributions of SLNs and SLN metastases in cervical and endometrial cancer with no false negative SLNs. Provided adherence to an anatomically defined algorithm sensitivity results for a SLN concept in endometrial and cervical cancer can be pooled. Hence, an SLN concept can be implemented in cervical cancer patients.

\section{Diagnostics}

\section{AN EVALUATION OF THE PERFORMANCE OF MOLECULAR ASSAYS TO IDENTIFY HOMOLOGOUS RECOMBINATION DEFICIENCY-POSITIVE TUMOURS IN OVARIAN CANCER}

${ }^{1} \mathrm{~W}$ Weichert ${ }^{*},{ }^{2} \mathrm{~N}$ Lukashchuk, ${ }^{2} \mathrm{~A}$ Yarunin, ${ }^{2} \mathrm{~L}$ Riva, ${ }^{2} \mathrm{~A}$ Easter, ${ }^{2} \mathrm{H}$ Bannister, ${ }^{3} \mathrm{P}$ Qiu, ${ }^{2} \mathrm{~T}$ French. ${ }^{1}$ Institute of Pathology, Technical University Munich, Munich, Germany; ${ }^{2}$ AstraZeneca, Cambridge, UK; ${ }^{3}$ Merck and Co., Inc., Kenilworth, USA

10.1136/ijgc-2021-ESGO.645

Introduction/Background* Homologous recombination deficiency (HRD), resulting from defects in the homologous recombination repair pathway, is a common feature of highgrade serous (HGS) ovarian cancer (OC) and predictive of sensitivity to PARP inhibitors. Developing reliable methods to determine HRD status of tumours is important to optimise clinical benefits of PARP inhibitors. Evaluation of HRD by genomic instability or BRCA1 and/or BRCA2 mutation
(BRCAm) is emerging as an important tool in OC. Here, we present a performance evaluation of two available molecular assays with potential to identify HRD-positive tumours by genomic instability in OC, compared with Myriad myChoice CDx (Myriad Genetic Laboratories, Inc.; US FDA-approved and EU CE-IVD marked).

Methodology Analytical performance of FoundationOne CDx (F1CDx; Foundation Medicine, Inc.), a US FDA-approved complementary diagnostic for genomic loss of heterozygosity in OC, and AmoyDx HRD Focus Panel (Amoy Diagnostics Co., Ltd) assays (both EU CE-IVD marked) were evaluated in a commercial clinical laboratory setting. Assay performance was evaluated as positive (PPA), negative (NPA) and overall (OPA) percent agreement with HRD status (determined by myChoice $\mathrm{CDx}$ ) of archival samples from patients with nongBRCAm platinum-sensitive HGS or endometrioid OC tumours. The assays determine genomic instability by different methodologies. Data are summarised descriptively.

Result(s)* Both F1CDx and AmoyDx HRD Focus Panel molecular HRD assays demonstrated analytical concordance with myChoice CDx, with differing levels of sensitivity and specificity at manufacturer-recommended cut-offs. F1CDx demonstrated the following agreements on genomic instability with myChoice CDx: PPA 67.6\%, NPA $85.7 \%$ and OPA $77.0 \%$ $(\mathrm{N}=148)$. AmoyDx HRD Focus Panel demonstrated the following agreements on genomic instability with myChoice CDx: PPA 92.0\%, NPA 52.1\% and OPA $72.4 \%$ using the existing algorithm and PPA 88.0\%, NPA 75.0\% and OPA $81.6 \%$ using a newly developed algorithm $(\mathrm{N}=98)$. Assay concordance for BRCAm detection was not undertaken because of limited BRCAm tumour samples.

Conclusion* F1CDx and AmoyDx HRD Focus Panel molecular HRD assays demonstrated concordance with, but not full equivalence to, myChoice CDx. Having multiple assays (providing their performance is adequate) to identify patients whose tumours harbour HRD is important to inform treatment decisions, as well as providing greater choice for clinicians and clinical laboratories.

\section{NEW INDEX BASED ON HUMAN EPIDIDYMIS PROTEIN 4 PERFORMS BETTER THAN RISK OF OVARIAN MALIGNANCY ALGORITHM IN PREMENOPAUSAL PATIENTS WITH PELVIC MASS}

\begin{abstract}
${ }^{1 ; 2} \mathrm{M}$ Katsyuba*${ }^{*}{ }^{1} \mathrm{R}$ Khasanov, ${ }^{3} \mathrm{~T}$ Madzhidov, ${ }^{4} \mathrm{G}$ Usmanova, ${ }^{2} \mathrm{~A}$ Akhmetzianova, ${ }^{5} \mathrm{G}$ Muratova, ${ }^{2} \mathrm{G}$ Fakhrutdinova. ${ }^{1}$ Kazan State Medical Academy - Branch Campus of the FSBEI FPE RMACPE MOH Russia, Oncology, Kazan, Russian Federation; ${ }^{2}$ Kazan Clinical Cancer Center, Gynecological Oncology, Kazan, Russian Federation; ${ }^{3}$ A.M. Butlerov Institute of Chemistry, Kazan Federal University, Chemistry, Kazan, Russian Federation; ${ }^{4}$ Kazan Clinical Cancer Center, Laboratory medicine, Kazan, Russian Federation; ${ }^{5}$ Kazan Federal University, International preparatory school, Kazan, Russian Federation
\end{abstract}

\subsection{6/ijgc-2021-ESG0.646}

Introduction/Background* Human epididymis protein 4 (HE4) has been reported as a promizing biomarker in the assessment of the risk of malignancy in patients, diagnosed with pelvic mass. Howewer, reference limits of HE4 do not provide clinically relevant discrimination between malignant and benign ovarian diseases. The clinical significance of well-known Risk of Ovarian Malignancy Algorithm (ROMA), which includes both HE4 and CA125, and its superiority over CA125 alone are still questionable. 
The aim of this study was to elaborate a new algorithm, based on serum CA125, HE4 and age, to assess the risk of malignancy in premenopausal patients with pelvic mass

Methodology The training dataset included 284 premenopausal patients operated because of presence of pelvic mass, out of which 35 and 249 had malignant and benign disease respectively. A novel algorithm, based on serum HE4, CA125 and patient's age as variables, has been developed by using the scenario of discrimination "benign diseases versus all stages of epithelial ovarian cancer (EOC) together with borderline ovarian tumors (BOT) FIGO stage 1c2-3c". This algorithm was named Risk of Ovarian Cancer Kazan Index (ROCK-I). The validating dataset consisted of consecutively operated premenopausal patients with pelvic mass out of which there were 187 cases of benign diseases, 20 EOC and 4 BOT FIGO stage $1 \mathrm{c} 2-3 \mathrm{c}$. An analysis with inclusion of BOT stage $1 \mathrm{a}-1 \mathrm{c} 1$ and non-EOC will be reported separately.

Result(s)* In the validating dataset the specificities of ROCK-I and ROMA were $92 \%$ and $85 \%$ respectively $(p<0.05)$. When the above-mentioned scenario of discrimination was used the sensitivities of ROCK-I and ROMA were 95.8\% and 91.7\% respectively, accuracies 92.4 and $85.8 \%$, positive predictive values $60.5 \%$ and $44 \%$ respectively. Areas under receiveroperating-characteristic curves (ROC-AUC) of ROCK-I, ROMA and CA125 were $0.984,0.94$ and 0.901 respectively. The difference in ROC-AUC between ROCK-I and CA125 was statistically significant $(p=0.03)$. A more detailed comparison of the performance of algorithms is shown in table 1 and figure 1 .

Abstract 1128 Table 1 Comparison of the performance of ROCKI and ROMA

\begin{tabular}{|c|c|c|c|c|c|c|c|c|}
\hline & $\begin{array}{l}\text { Sensitivity } \\
(95 \% \text { C1). } \%\end{array}$ & $\begin{array}{l}\text { Specificity } \\
(95 \% \text { CD) } \%\end{array}$ & $\begin{array}{c}\text { PPV } \\
(99 \% \text { CD). \% }\end{array}$ & $\begin{array}{l}\mathrm{NPV} \\
(99 \% \mathrm{CD}) \%\end{array}$ & $\begin{array}{l}\text { Accuracy } \\
\text { (95\%, CD).\% }\end{array}$ & $\begin{array}{c}\mathrm{LR+} \\
(95 \% \mathrm{CD})\end{array}$ & $\begin{array}{c}\text { DOR } \\
(95 \% \mathrm{CI})\end{array}$ & $\begin{array}{c}\text { ROC-AUC } \\
(99 \% \mathrm{CI})\end{array}$ \\
\hline & \multicolumn{8}{|c|}{$\begin{array}{l}\text { Training dataset } \\
\end{array}$} \\
\hline ROCK-I & $\begin{array}{c}89.6 \\
(75.96 .0)\end{array}$ & $\begin{array}{l}93.7^{*} \\
(90-96.1)\end{array}$ & $\begin{array}{l}66.7^{*} \\
(52.48)\end{array}$ & \begin{tabular}{|c|}
98.5 \\
$(96.0-99.4)$ \\
\end{tabular} & $\begin{array}{l}993.2^{*} \\
(89.7-6)\end{array}$ & $\begin{array}{l}14.3^{*} \\
(8.8-23.2)\end{array}$ & $\begin{array}{l}128.1 \\
(38.6-424.7) \\
\end{array}$ & $\begin{array}{l}0.997 \\
(0.94-0.992)\end{array}$ \\
\hline \multirow[t]{2}{*}{ ROMA } & $\begin{array}{l}83.9 \\
(685-926)\end{array}$ & $85.7^{*}$ & $45.2^{*}$ & $\begin{array}{l}97.4 \\
(94,4-988)\end{array}$ & $\begin{array}{c}85.5 * \\
(809-891)\end{array}$ & $\begin{array}{c}5.9^{*} \\
(42-83)\end{array}$ & $\begin{array}{l}31.1 \\
(118-820)\end{array}$ & 0.941 \\
\hline & \multicolumn{8}{|c|}{$\begin{array}{c}\text { (94.4-9.8.8) } \\
\text { Validating dataset. }\end{array}$} \\
\hline ROCK-I & $\begin{array}{l}\text { (78.9-9.89.9) } \\
\text { (1) }\end{array}$ & $\begin{array}{l}92.0^{*} \\
(88.1-95.4)\end{array}$ & $\begin{array}{c}60.5 \\
(48.4-71.5)\end{array}$ & $\begin{array}{l}99.4 \\
(96.2-99.9)\end{array}$ & $\begin{array}{l}99.4 .4 \\
(88.0-95.0)\end{array}$ & $\begin{array}{l}12.0 \\
(7.3-19.0)\end{array}$ & $\begin{array}{l}203.7 \\
(33.3-2091.1)\end{array}$ & $\begin{array}{l}0.984 \\
(0.969-0.998)\end{array}$ \\
\hline ROMA & $\begin{array}{c}91.7 \\
(73.0-99.0)\end{array}$ & $\begin{array}{c}85.0^{*} \\
(79.1-88.8)\end{array}$ & $\begin{array}{l}(35.4-53.0) \\
44.0\end{array}$ & $\begin{array}{l}95.8 \\
(95.5-99.7)\end{array}$ & $\begin{array}{c}85.8 \\
(80.3-90.2)\end{array}$ & $(4.3-8.8)$ & $\begin{array}{l}62.5 \\
(13.3-280.6)\end{array}$ & $\begin{array}{c}0.94 \\
(0.882-0.996)\end{array}$ \\
\hline
\end{tabular}

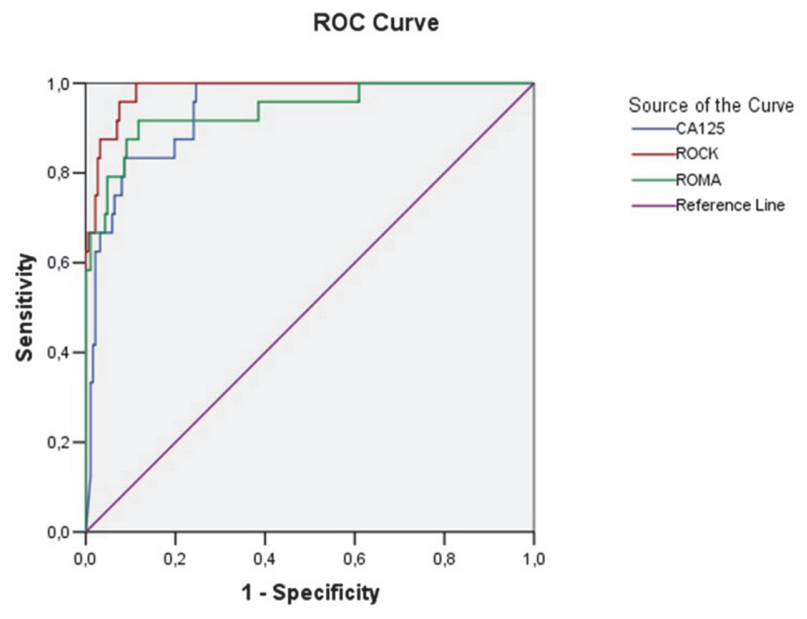

Abstract 1128 Figure 1 The ROC-curves of ROCK-I, ROMA and CA125 in the validating dataset
Conclusion* The proposed ROCK-I has demonstrated greater diagnostic performance than both ROMA and CA125 in the analyzed dataset. If an independent validation will show similar or even slightly lower difference between ROCK-I and ROMA it may provide a new basis of routine-use of HE4 in premenopausal patients with pelvic mass.

\section{UTERINE METASTASIS OF BREAST CANCER}

${ }^{1} \mathrm{D}$ Dhib*, ${ }^{2} Y$ Fertani, ${ }^{1} \mathrm{~F}$ Mghirbi, ${ }^{2} \mathrm{~L}$ Naija, ${ }^{1} \mathrm{~A}$ Gabsi, ${ }^{1} \mathrm{~A}$ Mokrani, ${ }^{1} \mathrm{~F}$ Ksontini, ${ }^{1} \mathrm{M}$ Ayadi, ${ }^{1} Y$ Yahyaoui, ${ }^{2} \mathrm{~K}$ Ben Ralah, ${ }^{1}$ A Mezlini. Institut Salah Azaiez, Medical Oncology, Tunis, Tunisia; ${ }^{2}$ Institut Salah Azaiez, Surgical Oncology, Tunis, Tunisia

\subsection{6/ijgc-2021-ESG0.647}

Introduction/Background* Uterine metastasis from extravaginal cancers is a rare event. A few cases of lobular invasive carcinoma with endometrial metastasis was reported in the litterature.

Result(s)* A 40 year old woman was treated in 2015 for a localised synchronous bilateral invasive ductal breast carcinoma. Human epidermal growth factor receptor (HER2) was amplified with positive endocrine receptor. She underwent in 2015 a neoadjuvant sequential hemotherapywith trastuzumab, followed by bilateral mastectomy, adjuvant trastuzumab and hormonotherapy (Tamoxifene with chemical ovarian suppression). In 2020, the CT scan showedliver, bone and peritoneal metastasis associated with a retro uterine mass suggesting a cervical cancer as a second cancer.

A biopsie was performed and the morphological feature with immunohistochemistry concluded to a metastasis of ductal breast carcinoma : Gata 3 protein(+), $\mathrm{ER}+, \mathrm{PR}+$, HER2 overexpressed and Wt1 negative .

Conclusion* Although rare, intrauterine breast cancer metastasis can occur even with a ductal breast cancer and physicians must go for a biopsy with immunohistochemistry.

\section{Endometrial cancer}

\section{MULTIPLEX QPCR HOTSPOT TESTING OF PATHOGENIC POLE MUTATIONS: A RAPID, SIMPLE AND RELIABLE APPROACH FOR POLE ASSESSMENT IN ENDOMETRIAL CANCER}

${ }^{1}$ AS Van den Heerik*, ${ }^{2} \mathrm{~N}$ Ter Haar, ${ }^{1} \mathrm{~N}$ Horeweg, ${ }^{2} \mathrm{~T}$ Van Wezel, ${ }^{1} \mathrm{C}$ Creutzberg, ${ }^{2} \mathrm{~V}$ Smit, ${ }^{2} \mathrm{~T}$ Bosse. 'Leiden University Medical Center (LUMC), Radiation Oncology, Leiden, Netherlands; 'Leiden University Medical Center (LUMC), Pathology, Leiden, Netherlands

\subsection{6/ijgc-2021-ESGO.648}

Introduction/Background* Detection of pathogenic somatic variants in the exonuclease domain (EDM) of the POLE-gene is of prognostic importance because of the excellent clinical outcomes of POLE-mutated endometrial cancers (EC). It is hypothesised that patients with POLE-mutated EC benefit of treatment de-escalation. Since $8-10 \%$ of EC carry a pathogenic POLE-mutation, it is of strong clinical importance to accurately determine the presence of these mutations. In current practice $P O L E$-status can only be determined by DNAsequencing methods, e.g. Sanger or Next-Generation-Sequencing (NGS). These techniques require a molecular biologist for 